Simon Hartlieb*, Michael Tscherpel, Flavio Guerra, Tobias Haist, Wolfgang Osten, Michael Ringkowski und Oliver Sawodny

\title{
Hochgenaue Kalibrierung eines holografischen Multi-Punkt-Positionsmesssystems
}

\author{
Highly accurate calibration of a holographic multipoint position measurement
}

https://doi.org/10.1515/teme-2019-0153

Eingang 24. Oktober 2019; angenommen 11. Dezember 2019

Zusammenfassung: Mit einem Multi-Punkt-Positionsmesssystem ist es möglich, Positionen und Orientierungen mit sehr hoher Genauigkeit bildbasiert zu messen. Das Messsystem besteht aus einem einfachen Kamerasystem, das um ein diffraktives optisches Element (DOE) erweitert wird. Durch die Multi-Punkt-Methode (MPM) werden Messunsicherheiten von deutlich unter 1/100 Pixel erzielt. Um dies in der Praxis anwenden zu können, ist eine entsprechend hochgenaue Kalibrierung nötig. In diesem Beitrag werden verschiedene Kalibrierverfahren und -funktionen vorgestellt und untersucht. Die Auswertung zeigt, dass sich zur Kalibrierung ein allgemeiner Polynomansatz zusammen mit der Nanomess- und Positioniermaschine NPMM-200 am besten eignet. Es werden Standardabweichungen des Restfehlers von $0,31 \mu \mathrm{m}$ im Objektraum (etwa 6/1000 Pixel im Bildraum) auf einer Fläche von etwa $87 \mathrm{~mm} \times 49 \mathrm{~mm}$ erreicht (Polynomgrad $=9$ ).

Schlagwörter: Kalibrierung, Positionsmessung, Bildverarbeitung, Multipoint, computer-generierte Hologramme, Telezentrie, Nanomess- und Positioniermaschine.

Abstract: A multipoint position measurement system is an image based measurement system to detect positions and orientations with very high accuracy. It consists of a simple camera system that is extended by a diffractive optical element (DOE) to reach accuracies of less than 1/100 pixel. To use this method in practice, an accurate calibration is necessary. In this artircle we will introduce different calibration methods and -functions and investigate them. It is to be seen that calibration with a general polynomial in combination with the Nanomeasurement- and positioning machine NPMM-200 provides the necessary

\footnotetext{
*Korrespondenzautor: Simon Hartlieb, Institut für Technische Optik, Universität Stuttgart, Pfaffenwaldring 9, 70569 Stuttgart, Germany, E-Mail: hartlieb@ito.uni-stuttgart.de

Michael Tscherpel, Flavio Guerra, Tobias Haist, Wolfgang Osten, Institut für Technische Optik, Universität Stuttgart, Pfaffenwaldring 9, 70569 Stuttgart, Germany

Michael Ringkowski, Oliver Sawodny, Institut für Systemdynamik, Universität Stuttgart, Waldburgstr. 19, 70563 Stuttgart, Germany
}

means to reach standard deviations in reprojection error of $0,31 \mu \mathrm{m}$ in object space (approximately 6/1000 pixel in image space) in an area of $87 \mathrm{~mm} \times 49 \mathrm{~mm}$ (polynomial or$\operatorname{der}=9$ ).

Keywords: Calibration, position measurement, image processing, multipoint, computer generated hologram, telecentric systems, nanomeasuring and nanopositioning machine.

\section{Einleitung und Stand der Technik}

\subsection{Einleitung}

In vielen industriellen Anwendungen spielt die hochgenaue Messung von Positionen eine bedeutende Rolle. Für Werkzeug- beziehungsweise Koordinatenmessmaschinen ist vor allem die Relativposition zwischen dem Bearbeitungskopf (TCP) und einem zu bearbeitenden Werkstück (WP) von Interesse. In der Regel werden in konventionellen Systemen die Positionen des TCP indirekt über Messsysteme in den Verfahrachsen (z. B. interferometrische Messung, Glasmaßstab) ermittelt. Dabei wird von ideal starren Körpern ausgegangen. Kurze Prozesszeiten haben oft hohe Beschleunigungen zur Folge, die in Kombination mit großen bewegten Massen zu einer Abweichung zwischen der von der Maschine ermittelten und der realen Relativposition führt.

In Abbildung 1 ist ein einfaches Verfahren zur direkten Messung der Relativposition dargestellt. Es werden sowohl am Werkzeug als auch am Werkstück Leuchtmarker platziert und über zwei Kamerasysteme abgebildet.

Somit kann im Bild die Relativposition zwischen beiden Leuchtmarkern direkt bestimmt werden. In der Praxis ist nach dieser Methode die Position eines Leuchtmarkers mit einer RMS-Abweichung (quadratischer Mittelwert) von etwa 1/100 Pixel detektierbar [1]. Für die Anwendung in einem Messsystem muss durch eine Kalibrierung der $\mathrm{Zu}$ sammenhang zwischen dem Bildraum und dem Objektraum hergestellt werden. Mit einer Kalibrierung nach dem 


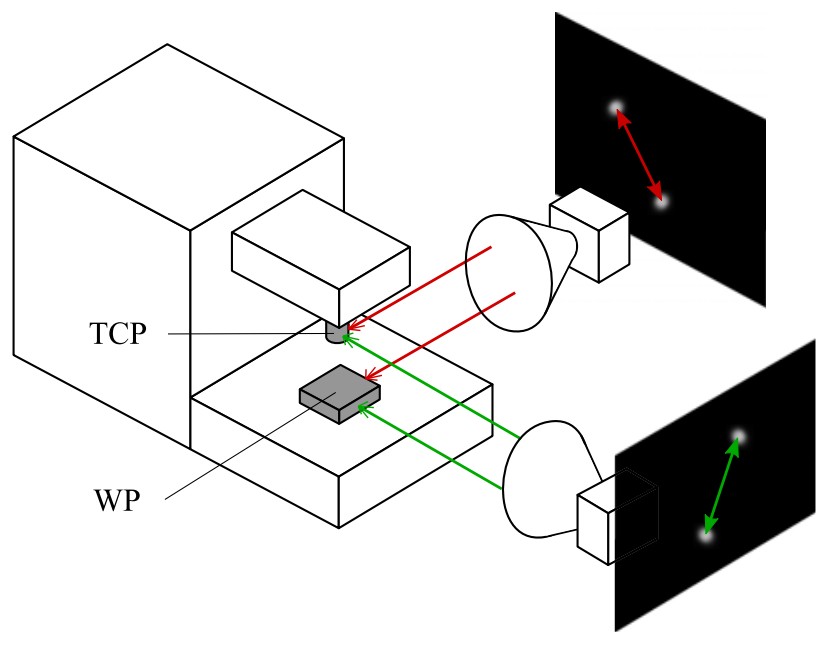

Abb. 1: Bildbasierte Messung der Relativposition zwischen Werkzeug (TCP) und Werkstück (WP) in einer Werkzeugmaschine.

aktuellen Stand der Technik verringert sich die Genauigkeit der Positionsbestimmung je nach Kalibrierung auf etwa 0,05 Pixel, was im Objektraum etwa $3 \mu \mathrm{m}$ entspricht (Abbildungsmaßstab $=0,1$ und Pixelgröße $=5,86 \mu \mathrm{m}$ ). Hochgenaue Bearbeitungs- und Messanwendungen wie beispielsweise die Präzisionsoberflächenbearbeitung von Optiken oder die Vermessung von Asphären verlangen jedoch deutlich geringere Messunsicherheiten. Die MPM zusammen mit einer entsprechend genauen Kalibrierung bietet die Möglichkeit, diese Genauigkeit erheblich zu verbessern.

Die Idee der MPM ist es, einen einzelnen leuchtenden Objektpunkt auf ein Muster von $N$ Bildpunkten auf dem Kamerasensor abzubilden, um so durch anschließende Mittelung die Genauigkeit der Positionsdetektion zu steigern. Das vordefinierte Muster wird durch ein diffraktives optisches Element (DOE) erzeugt. In Abbildung 2 ist ein beidseitig telezentrisches Linsensystem mit einem DOE in

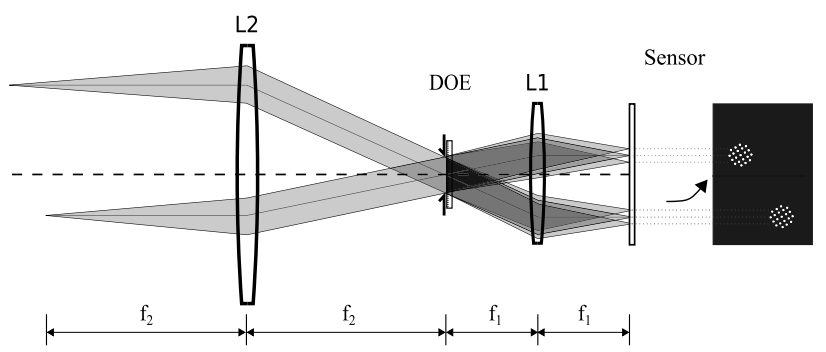

Abb. 2: Telezentrisches Linsensystem (L1 und L2) mit diffraktivem optischen Element (DOE) in der Blendenebene zur Erzeugung der Multispotmuster. der Blendenebene dargestellt. Ein einzelner Lichtpunkt wird in der Abbildung durch das DOE in ein Muster von $N=21$ Spots repliziert. Da Störeinflüsse wie Photonenrauschen, Diskretisierung oder Sensorrauschen zwischen den Replikationen unkorreliert sind, verbessert eine Mittelung über alle Spotschwerpunkte die Detektionsgenauigkeit im Idealfall um den Faktor $\sqrt{N}$. Die RMS-Abweichung von der exakten Position konnte in experimentellen Untersuchungen von 0,01 Pixel auf 0,0028 Pixel für $N=16$ verbessert werden [2]. Für eine theoretisch ideale Kalibrierung wäre somit ein bildbasiertes Messsystem mit einer objektseitigen Genauigkeit von $0,164 \mu \mathrm{m}$ möglich.

Die mit der MPM erzielbare extrem geringe (statistische) Messunsicherheit verlangt für absolut messende Anwendungen nach einer entsprechend genauen Kalibrierung. Gängige Kamerakalibrierungen (siehe z. B. [3-6]) sind beim Stand der Technik nicht in diesem Genauigkeitsbereich realisiert.

Im Folgenden werden wir entsprechende Verzeichnungsmodelle darstellen und experimentelle Ergebnisse der hochgenauen Kalibrierung eines telezentrischen Objektivs mit der Nanomess- und Positioniermaschine NPMM-200 (siehe Abschnitt 4.1) vorstellen.

\subsection{Telezentrisches Kameramodell}

Typische beidseitig telezentrische Objektive bestehen in der Regel aus zwei Linsengruppen. Die Aperturblende ist in der gemeinsamen Brennebene der beiden Gruppen (siehe Abb. 2). Hierdurch wird sichergestellt, dass die Schwerstrahlen der Abbildung sowohl objektseitig wie auch bildseitig für alle Feldpunkte parallel zur optischen Achse verlaufen. Der Abbildungsmaßstab bleibt somit bei Objektverschiebung konstant (objektseitige Telezentrie) und die am Bildsensor ankommende Strahlungsverteilung ist für jeden Feldpunkt gleich orientiert (bildseitige Telezentrie). Dadurch nehmen die Mikrolinsen vor den Pixeln keinen Einfluss auf die Lichtverteilung auf dem Sensor. Im Umfeld der hochgenauen bildbasierten Messtechnik werden aus diesen Gründen häufig beidseitig telezentrische $\mathrm{Ob}$ jektive verwendet. Da in diesem Beitrag höchste Genauigkeiten angestrebt werden, wurde ein beidseitig telezentrisches Kamerasystem zur Abbildung gewählt. Es sei jedoch darauf hingewiesen, dass die MPM gleichfalls in entozentrischen Systemen einsetzbar ist [7].

Die gesamte verzeichnungsfreie telezentrische $\mathrm{Ab}$ bildung eines Objektpunktes $P=\left[\begin{array}{lll}X_{W} & Y_{W} & Z_{W}\end{array}\right]^{T}$ vom Weltkoordinatensystem auf die unverzeichneten Bildkoordinaten $\left[x_{u}, y_{u}\right]$ im Sensorkoordinatensystem ergibt sich 
$\mathrm{zu}[8]$

$$
\left[\begin{array}{c}
x_{u} \\
y_{u} \\
1
\end{array}\right]=\underbrace{\left[\begin{array}{ccc}
\frac{m}{d u} & 0 & u_{0} \\
0 & \frac{m}{d v} & v_{0} \\
0 & 0 & 1
\end{array}\right]}_{\text {intrinsisch }} \underbrace{\left[\begin{array}{cccc}
r_{11} & r_{12} & r_{13} & t_{x} \\
r_{21} & r_{22} & r_{23} & t_{y} \\
0 & 0 & 0 & 1
\end{array}\right]}_{\text {extrinsisch }}\left[\begin{array}{c}
X_{W} \\
Y_{W} \\
Z_{W} \\
1
\end{array}\right]
$$

Die extrinsische Kalibriermatrix beschreibt die Beziehung zwischen dem Welt- und dem Kamerakoordinatensystem, wobei $R=\left[r_{i j}\right]$ die Rotationsmatrix und $T=\left[\begin{array}{ll}t_{x} & t_{y}\end{array}\right]^{T}$ der Translationsvektor zwischen diesen Koordinatensystemen ist.

Die intrinsische Kameramatrix beschreibt die ideale Abbildung bzw. Projektion des Punktes $P$ auf die Bildebene. Darin sind $m$ die Vergrößerung und $d u, d v$ die Pixelgrößen in $x$ - bzw. $y$-Richtung des Sensors. Der Versatz zwischen dem Sensorkoordinatenursprung und der Bildmitte wird durch $u_{0}$ und $v_{0}$ berücksichtigt. Da ein telezentrisches Objektiv im idealen Fall eine perfekte Parallelprojektion ausführt, besitzt die Tiefenkomponente $Z_{W}$ keinen Einfluss auf die Bildposition [9].

Ein reales telezentrisches Abbildungssystem besitzt eine Verzeichnung, welche durch Addition der Fehlerfunktionen $\left(\delta_{x}, \delta_{y}\right)$ auf die unverzeichneten Koordinaten $\left[x_{u}, y_{u}\right]$ berücksichtigt wird:

$$
\left[\begin{array}{l}
x_{d} \\
y_{d}
\end{array}\right]=\left[\begin{array}{l}
x_{u} \\
y_{u}
\end{array}\right]+\left[\begin{array}{l}
\delta_{x}\left(x_{u}, y_{u}\right) \\
\delta_{y}\left(x_{u}, y_{u}\right)
\end{array}\right]
$$

Bei einem Objektiv mit Telezentriefehler ergibt sich eine Variation der Parameter der intrinsischen Matrix und - in geringem Maß - der Verzeichnung, so dass Gleichungen (1) und (2) für geeignete Ebenen zu betrachten sind. Ein gängiges Verfahren um Verzeichnungen im Kamerasystem zu korrigieren besteht darin, sie durch ein passendes Verzeichnungsmodell in Form eines Polynoms anzunähern.

\subsection{Kalibrierung und Verzeichnungsmodelle}

Für eine Kamerakalibrierung wird häufig ein bekanntes, planares Kalibriertarget in unterschiedlichen Ausrichtungen und Positionen vom Kamerasystem aufgenommen [10-13]. Die bekannte Geometrie des Targets wird durch das optische System fehlerbehaftet ("verzeichnet”) abgebildet. Eine Korrektur kann nach der Bildaufnahme durch geeignete Kalibrierfunktionen erreicht werden, wobei zwei unterschiedliche Modelle zur Anwendung kommen [14]. Im physikalischen Modell wird die Ursache der Verzeichnung untersucht und deren Auswirkungen durch speziell darauf angepasste Korrekturterme kompensiert.
Im Gegensatz dazu wird im phänomenologischen Modell der Effekt der Verzeichnung unabhängig von dessen Ursache korrigiert [14]. Beide Modelle werden nachfolgend kurz vorgestellt.

\section{Physikalisches Modell}

Bei diesem Modell werden üblicherweise die Verzeichnungsterme der Radial- $\left(\delta_{x r}\right)$, Dezentrierungs- $\left(\delta_{x d}\right)$ und Prismenkeilverzeichnung $\left(\delta_{x p}\right)$ betrachtet $[3,4,15,16]$. Die Fehler $\left(\delta_{x}, \delta_{y}\right)$ aus Gleichung (2) ergeben sich folglich aus der Summe dieser Terme

$$
\begin{aligned}
& \delta_{x}\left(x_{u}, y_{u}\right)=\delta_{x r}+\delta_{x d}+\delta_{x p} \\
& \delta_{y}\left(x_{u}, y_{u}\right)=\delta_{y r}+\delta_{y d}+\delta_{y p} .
\end{aligned}
$$

Drückt man die von Weng u. a. [15] beschriebenen Terme in kartesischen Koordinaten aus so erhält man für die radiale Verzeichnung

$$
\begin{aligned}
& \delta_{x r}=k_{1} x_{u}\left(x_{u}^{2}+y_{u}^{2}\right)+k_{2} x_{u}\left(x_{u}^{2}+y_{u}^{2}\right)^{2}+\cdots \\
& \delta_{y r}=k_{1} y_{u}\left(x_{u}^{2}+y_{u}^{2}\right)+k_{2} y_{u}\left(x_{u}^{2}+y_{u}^{2}\right)^{2}+\cdots
\end{aligned}
$$

Dabei sind $k_{1}, k_{2}, \ldots$ die Koeffizienten der radialen Verzeichnung.

Die Prismenkeilverzeichnung kann durch eine Verkippung einzelner Linsen oder des Sensors beim Einbau verursacht werden. Mathematisch wird sie durch das Hinzufügen eines Prismenkeils modelliert. Es entstehen sowohl radiale als auch tangentiale Verzeichnungen, die Terme ergeben sich zu

$$
\begin{aligned}
& \delta_{x p}=-\sin \varphi_{1}\left[i_{1}\left(x_{u}^{2}+y_{u}^{2}\right)+i_{2}\left(x_{u}^{2}+y_{u}^{2}\right)^{2}+\cdots\right] \\
& \delta_{y p}=\cos \varphi_{1}\left[i_{1}\left(x_{u}^{2}+y_{u}^{2}\right)+i_{2}\left(x_{u}^{2}+y_{u}^{2}\right)^{2}+\cdots\right] .
\end{aligned}
$$

Der Winkel $\varphi_{1}$ liegt zwischen der X-Achse des Kamerakoordinatensystems und der Achse maximaler tangentialer Verzeichnung; $i_{1}, i_{2}$ sind die Koeffizienten der Prismenkeilverzeichnung.

Die Terme der Dezentrierungsverzeichnung werden aus Platzgründen hier nicht aufgeführt, können jedoch in [15] nachgeschlagen werden.

Sowohl von Zollner u. a. [17] als auch von Salvi u.a. [18] wurden unterschiedliche physikalische Modelle untersucht und verglichen. Dabei schneidet die vorgestellte Methode nach Weng u.a. [15] mit einer Genauigkeit von 0,044 Pixel am besten ab. Sie wird z. B. in [4] angewendet, wobei eine Kalibriergenauigkeit von 0,052 Pixel erreicht wurde. 


\section{Phänomenologisches Modell}

Die verzeichnete Position jedes Lichtpunktes wird bei diesem Ansatz durch eine Potenzreihe der Form

$$
\begin{aligned}
& x_{d}=\sum_{i=0}^{n_{\text {order }}} \sum_{j=0}^{n_{\text {order }}} L_{i j} X_{W}^{i} Y_{W}^{j} \\
& y_{d}=\sum_{i=0}^{n_{\text {order }}} \sum_{j=0}^{n_{\text {order }}} M_{i j} X_{W}^{i} Y_{W}^{j}
\end{aligned}
$$

dargestellt. Dabei ist $n_{\text {order }}$ der Grad des Polynoms, $L_{i j}$ und $M_{i j}$ sind die Koeffizientenmatrizen der Potenzreihen für $x_{d}$ bzw. $y_{d}$. Das Polynom ersetzt die externe und interne Kalibriermatrix aus Gleichung (1) und bildet die Lichtpunktposition direkt vom Weltkoordinatensystem auf den Bildsensor ab.

Tang u.a. haben in [5] den physikalischen und phänomenologischen Kalibrieransatz verglichen. Dabei ergab sich ein durchschnittlicher Restfehler von 0,03 Pixel für den physikalischen Ansatz und 0,04 Pixel für den phänomenologischen Ansatz (70 mm Objektiv, 9. Ordnung). In dem Artikel wird darauf hingewiesen, dass der Restfehler von 0,03 bis 0,04 Pixel etwa der Detektionsgenauigkeit von Eckpunkten entspricht. Somit liefern in diesem Genauigkeitsbereich beide Ansätze eine nahezu ideale Korrektur der untersuchten Objektive.

\section{Kalibriermethode}

Beim Stand der Technik werden heute Kamerakalibrierungen in der Regel mittels spezieller Kalibriertargets (z. B. Schachbrett) durchgeführt. Für die hier notwendige hochgenaue Kalibrierung sind entsprechende Standardverfahren nicht genau genug, da die Positionsbestimmung des einzelnen Kalibrierpunkts (z. B. Ecke Schachfeld) nicht die erforderliche Genauigkeit erzielt. Es ist also bereits bei der Kalibrierung die MPM anzusetzen und mit Lichtquellen zu arbeiten. Hierbei können entweder viele Quellen (z. B. Lochblendenarray) oder scannend eine Quelle verwendet werden.

\subsection{Kalibriertarget}

Als Kalibriertarget kann eine beleuchtete Lochmaske verwendet werden, deren Lochabstände so groß sind, dass sich die Multispotmuster auf dem Bildsensor nicht überlagern. Für die Beurteilung der Eignung dieser Methode werden folgende Fehlereinflüsse berücksichtigt und mit Hilfe von Simulationen untersucht:
1. Beleuchtung der Lochmaske

2. Toleranzen der Maskenlochposition

3. Größe eines Maskenlochs

Als Simulationsmodell wird das Zemax ${ }^{\circledR}$-Modell des im Messaufbau verwendeten telezentrischen Objektivs (DTCM110-150-AL) verwendet.

1. Beleuchtung: Eine generelle Herausforderung bei der Beleuchtung besteht darin, jeden Lichtpunkt der Lochmaske möglichst gleich auszuleuchten, um die mittels Grauwertschwerpunkt errechneten Positionen nicht zu verfälschen.

In Abbildung 3 sind drei mögliche Beleuchtungsarten dargestellt. Eine kollimierte Beleuchtung der lithografisch hergestellten Lochmaske wäre denkbar, ist jedoch sehr ineffizient, da ein Großteil des Lichts von der Maske reflektiert bzw. absorbiert wird. Ein Mikrolinsenarray vor der Lochmaske (vgl. Abb. 3 a)) verbessert die Lichtausbeute. Im Vergleich dazu, wird in Abb. 3 b) die Lochmaske in einem $2 f$-Aufbau mittels Streuscheibe beleuchtet. Die Streuscheibe wird gewählt, um eine möglichst gleichmäßige Abstrahlcharakteristik jedes Lichtpunktes auf der Lochmaske zu erzeugen. In der dritten Beleuchtungsvariante (Abb. $3 \mathrm{c})$ ) wird jedes Maskenloch durch eine eigene Lichtquelle (z. B. LED) beleuchtet.

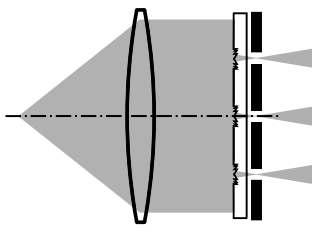

a)

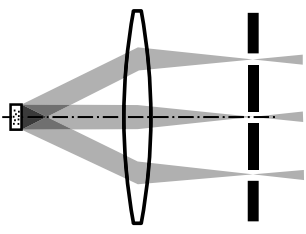

b)

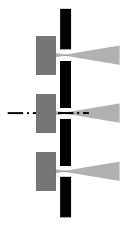

c)
Abb. 3: Mögliche Beleuchtungen der Lochmaske. a) Kollimiert auf Mikrolinsenarray b) $2 f$-Aufbau mit Streuscheibe c) Lichtquelle direkt vor Maskenloch.

Durch die Simulation stellte sich heraus, dass bei den ersten beiden Varianten die Linse zur Beleuchtung des Mikrolinsenarrays bzw. der Lochmaske problematisch ist. Im Idealfall sollte so beleuchtet werden, dass an jedem Maskenloch der Hauptstrahl parallel zur optischen Achse austritt, um die Schwerpunktposition auf dem Sensor nicht zu verfälschen. Dies wird nur bei einer ideal kollimierten Beleuchtung erreicht. Bei Kataloglinsen führen Aberrationen dazu, dass die Hauptstrahlen im Feld nicht mehr parallel sind. Wie sich der Abstrahlwinkel $\Psi$ auf die Spotposition $y^{\prime}$ auswirkt, ist in Abbildung 4 dargestellt. In der Zemax ${ }^{\circledR}$-Simulation wurde ein Maskenloch 


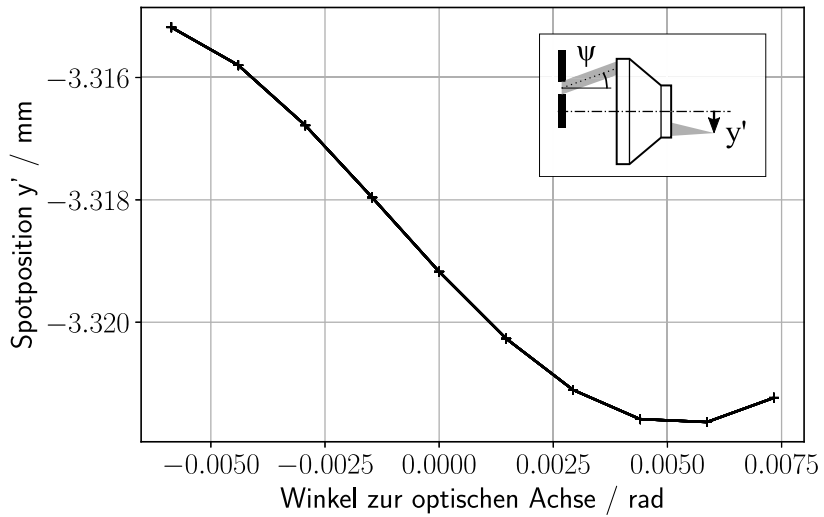

Abb. 4: Simulation des Zusammenhangs zwischen Hauptstrahlwinkel $\Psi$ und Spotposition $y^{\prime}$.

bei $y=30 \mathrm{~mm}$ mit paraxial kollimierten Lichtstrahlen unterschiedlicher Winkel $\Psi$ beleuchtet. Die Spots der jeweiligen Strahlen werden durch das Simulationsmodell auf unterschiedliche Positionen $y^{\prime}$ auf dem Detektor abgebildet. Die Auftreffpositionen schwanken über 6,5 $\mu \mathrm{m}$. Zur Positionsmessung wird der Schwerpunkt dieser Verteilung auf dem Bildsensor ermittelt. Somit genügt ein Intensitätsunterschied von $1 \%$ zwischen zwei Strahlbündeln die mit +5 mrad bzw. -5 mrad vom Maskenloch abstrahlen, um den Schwerpunkt auf dem Sensor um 13,4 nm zu verschieben. Bei einer Pixelgröße von 5,86 $\mu \mathrm{m}$ entspricht dies etwa 2/1000 Pixel. Dieser Fehlereinfluss würde somit bereits im Bereich der angestrebten Genauigkeit liegen. Ein aufwändiges telezentrisches Beleuchtungssystem könnte eingesetzt werden, vermeidet aber nicht die Problematik der Abhängigkeit von der räumlichen Abstrahlcharakteristik der Lichtquelle.

Die dritte Beleuchtungsvariante vermeidet diese Nachteile der beiden anderen Varianten. Allerdings ist sie sehr aufwändig in der Umsetzung, da jedes Maskenloch eine eigene aktive Lichtquelle benötigt, deren Ausrichtung, Abstrahlcharakteristik sowie Intensitätsverteilung möglichst gleich zu den anderen sein muss. Außerdem wirkt sich der Wärmeeintrag jeder Lichtquelle auf die Kalibriergeometrie aus. Es wurde ebenfalls die Verwendung eines Displays erwogen, auf dem die Lichtquellen der Lochmaske über einzelne leuchtende Pixel erzeugt werden. Aufgrund der Strahlvervielfältigung durch das Hologramm müsste in diesem Fall allerdings die Belichtungszeit der Kamera deutlich erhöht werden, wodurch sich das SignalRausch-Verhältnis verschlechtert und somit der Vorteil der MPM verloren geht.

2. Toleranzen: Die Positioniergenauigkeit einzelner Löcher in einer lithografisch geschriebenen Chrommaske liegt, für eine große Maske im Bereich $1 \mu \mathrm{m}$, was einem bildseitigen Fehler von 0,017 Pixel entspricht. Der Restfehler wird somit im Mittel selbst mit einer idealen Kalibrierfunktion immer von dieser Positionsgenauigkeit der einzelnen Maskenlöcher abhängen. Durch Messung der exakten Lage könnte dieser Wert weiter verbessert werden, der Aufwand hierfür wäre allerdings beträchtlich.

3. Größe: Um den Einfluss der Lochgröße auf den Schwerpunkt in der Sensorebene zu charakterisieren, muss die Lochmaske perfekt gleichmäßig ausgeleuchtet sein. Hierzu wurde in der Simulation das Maskenloch von einer kollimiert beleuchteten, nach dem LambertGesetz streuenden Kreisfläche beleuchtet. Dies entspricht einer theoretisch perfekt gleichmäßigen Ausleuchtung. Um die Schwerpunktposition in dieser Genauigkeit angeben zu können, wurde Raytracing mit $\geq 10^{6}$ Strahlen bei unterschiedlichen Größen der Lichtquelle durchgeführt. Die Abhängigkeiten des Schwerpunkts vom Lochradius sind in Tabelle 1 angegeben. Die Schwerpunktverschiebung zwischen den Lochradien $1 \mu \mathrm{m}$ und $50 \mu \mathrm{m}$ beträgt $12 \mathrm{~nm}$, was ebenfalls etwa 2/1000 Pixel auf dem Sensor entspricht. Somit sollten die zur Kalibrierung und die zur Messung eingesetzte Lichtquelle möglichst die gleiche Größe haben. Die unterschiedliche Schwerpunktposition ist auf die Verzeichnung des Objektivs zurückzuführen. Je größer die Lichtquelle ist, desto unterschiedlicher sind die Wege der Randstrahlen durch das Objektiv, was zu unterschiedlichen Verzeichnungen führt.

Tab. 1: Schwerpunktposition $y^{\prime}$ in Abhängigkeit der MaskenlochgröBe bei $y=30 \mathrm{~mm}$.

\begin{tabular}{rrr}
\hline Radius $\boldsymbol{R}$ & Position $\boldsymbol{y}^{\prime}$ & Differenz zu $\boldsymbol{R}=\mathbf{1} \boldsymbol{\mu \mathrm { m }}$ \\
\hline $1 \mu \mathrm{m}$ & $3608,5834 \mu \mathrm{m}$ & - \\
$10 \mu \mathrm{m}$ & $3608,5765 \mu \mathrm{m}$ & $6,9 \mathrm{~nm}$ \\
$50 \mu \mathrm{m}$ & $3608,5714 \mu \mathrm{m}$ & $12 \mathrm{~nm}$ \\
\hline
\end{tabular}

\subsection{Positioniermaschine}

Statt einer Lochmaske ist es ebenfalls möglich, jedes Maskenloch durch eine nacheinander an jede dieser Stellen positionierte Einzellichtquelle zu ersetzen. Zum Verfahren der Lichtquelle kann eine hochgenaue Positioniermaschine wie beispielsweise die Nanomess- und Positioniermaschine NPMM-200 [19] verwendet werden. Nachteilig an dieser Variante ist, dass keine "Single-Shot"-Kalibrierung möglich ist. Das mäanderförmige Positionieren der Lichtquelle benötigt Zeit, wodurch wechselnde Umgebungsbedingungen das Kalibrierergebnis verfälschen können. Weiterhin hängt das Kalibrierergebnis mitunter von der 
Genauigkeit der zur Verfügung stehenden Positioniermaschine ab.

Von Vorteil ist, dass zur Kalibrierung und in der Messanwendung dieselbe Lichtquelle eingesetzt werden kann. Positionsfehler auf dem Sensor, die durch unterschiedliche Abstrahlcharakteristik und Intensitätsverteilung auftreten, können so reduziert werden. Außerdem kann das Raster, auf dem die Lichtquelle verfahren wird, beliebig fein gewählt und so die Anzahl der Stützstellen für die Polynomoptimierung erhöht werden. Aufgrund der Untersuchungen des vorangegangenen Abschnitts wird zur Kalibrierung eine Positioniermaschine eingesetzt. Nachfolgend wird geklärt, welche Kalibrierfunktion zur Modellierung der Verzeichnung zu wählen ist.

\section{Kalibrierfunktion}

Um eine geeignete Kalibrierfunktion $\mathrm{zu}$ finden, wird mittels Raytracing ein einfaches telezentrisches Linsensystem (Objektfeld $=17,67 \mathrm{~mm}, N A=0,00167, \beta^{\prime}=0,1$ ) untersucht. Um Fertigungsfehler zu simulieren wird die bildseitige Linse um $1 \mathrm{~mm}$ dezentriert und um $1^{\circ}$ zur optischen Achse verkippt. Anschließend werden für $100 \times 100$ Punkte im Objektraum die dazugehörigen verzeichneten Bildpunkte der Hauptstrahlen bestimmt.

Beim physikalischen Ansatz entfällt das Optimierungsproblem bezüglich der Rotationsmatrix $R$ und des Translationsvektors $T$ ebenso wie die Verschiebung vom Bildkoordinatensystem in das Sensorkoordinatensystem $u_{0}$ und $v_{0}$, da in der Raytracing Software nicht zwischen dem Welt- und dem Kamerakoordinatensystem unterschieden wird. Das telezentrische Kameramodell aus Gleichung (1) vereinfacht sich zu

$$
\left[\begin{array}{c}
x_{d} \\
y_{d} \\
1
\end{array}\right]=\left[\begin{array}{ccc}
m & 0 & \delta_{x} \\
0 & m & \delta_{y} \\
0 & 0 & 1
\end{array}\right]\left[\begin{array}{cccc}
1 & 0 & 0 & 0 \\
0 & 1 & 0 & 0 \\
0 & 0 & 0 & 1
\end{array}\right]\left[\begin{array}{c}
X_{W} \\
Y_{W} \\
Z_{W} \\
1
\end{array}\right] .
$$

Die intrinsischen Parameter sind $\delta_{x}\left(\varphi_{0}, \varphi_{1}, i_{i}, j_{i}, k_{i}\right)$, $\delta_{y}\left(\varphi_{0}, \varphi_{1}, i_{i}, j_{i}, k_{i}\right)$ sowie der Abbildungsmaßstab $m$.

Die Fehlerfunktion für die Least-Squares-Optimierung ergibt sich sowohl für das phänomenologische als auch das physikalische Modell zu

$$
F=\sum_{i=1}^{n}\left|p_{i}-\tilde{p}_{i}\right| .
$$

Dabei ist $p_{i}$ die Position des mittels Raytracing bestimmten Bildpunktes, $\tilde{p}_{i}$ die nach Gleichung (7) bzw. (6) berechnete Position desselben Bildpunktes.

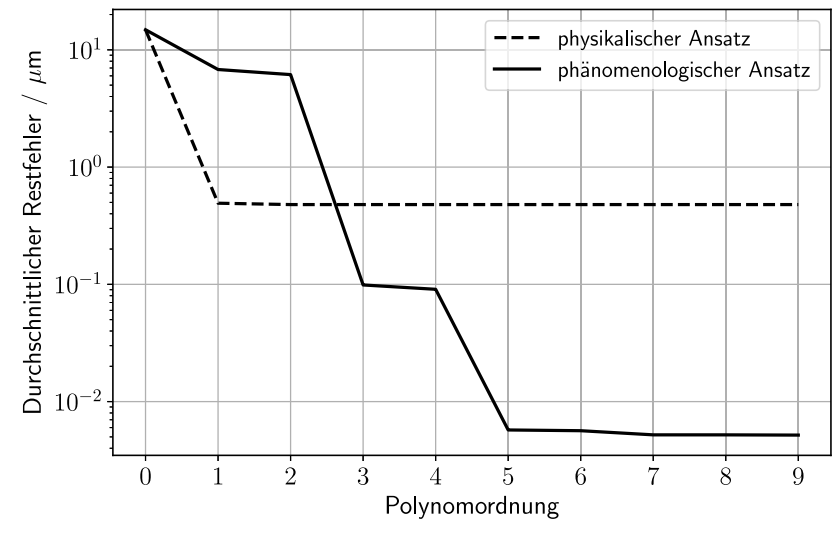

Abb. 5: Durchschnittlicher Restfehler beider Korrekturfunktionen.

Der durchschnittliche Restfehler in Abhängigkeit der Polynomordnung für beide Modelle ist in Abbildung 5 dargestellt. Mit den speziellen Korrekturtermen des physikalischen Modells lässt sich die anfängliche durchschnittliche Restverzeichnung bereits mit der ersten Ordnung aller drei Korrekturterme von $14,84 \mu \mathrm{m}$ auf 0,516 $\mu \mathrm{m}$ korrigieren. Jedoch bringt eine weitere Erhöhung der Terme keine weitere Verbesserung. Bei einer Pixelgröße von 5,86 $\mu \mathrm{m}$ kann mit diesem Kalibrieransatz demnach das untersuchte System auf etwa 0,088 Pixel Restfehler kalibriert werden.

Mit dem allgemeinen Polynomansatz des phänomenologischen Modells korrigiert ein Polynom 3. Ordnung die durchschnittliche Verzeichnung bereits auf $98,79 \mathrm{~nm}$. $\mathrm{Ab}$ der 5. Ordnung bereits auf 5,729 nm (etwa 1/1000 Pixel), wobei sich für höhere Ordnungen jedoch keine merkliche Verbesserung mehr ergibt. Die Positionsdaten in den Grid-Distortion-Tabellen werden auf fünf Nachkommastellen angegeben. Somit erfährt jeder Bildpunkt einen Rundungsfehler von durchschnittlich 2,5 nm, wodurch die weitere Korrektur der Verzeichnung limitiert wird.

Diese Art der Kalibrierung liefert somit eine theoretisch ausreichend genaue Verzeichnungskorrektur, selbst für ein stark verzeichnetes Ausgangssystem, wie es in dieser Simulation verwendet wurde.

\section{Kalibriermessungen}

In diesem Abschnitt wird der Kalibrieraufbau, die verwendeten Materialien und die Kalibrierergebnisse mit dem allgemeinen Polynomansatz beschrieben. Um das MultiPunkt-Messsystem zu kalibrieren wird eine Lichtquelle im Objektraum mäanderförmig an $M$ Messpunkte gefahren. Für das Bewegen der Lichtquelle wird die NPMM-200 genutzt. 


\subsection{Nanomess- und Positioniermaschine NPMM-200}

Die NPMM-200 besteht aus einem dreiachsigen Positioniersystem mit Präzisionsführungen und -antrieben. Sie ist nach dem sogenannten Sample Scanning Mode konstruiert, bei dem das Messobjekt und nicht der Messsensor bewegt wird. Die Position des verfahrbaren Messobjektträgers wird über drei Interferometer bestimmt. Die Interferometer sind so angeordnet, dass sich der virtuelle Schnittpunkt ihrer Strahlen raumfest im Antastpunkt des Messsensors befindet. Der bewegliche Messobjektträger besitzt drei hochgenau vermessene spiegelnde Flächen [20], an denen die Interferometerstrahlen reflektiert werden. In Abbildung 6 ist der Messobjektträger und der metrologische Rahmen der NPMM-200 dargestellt.

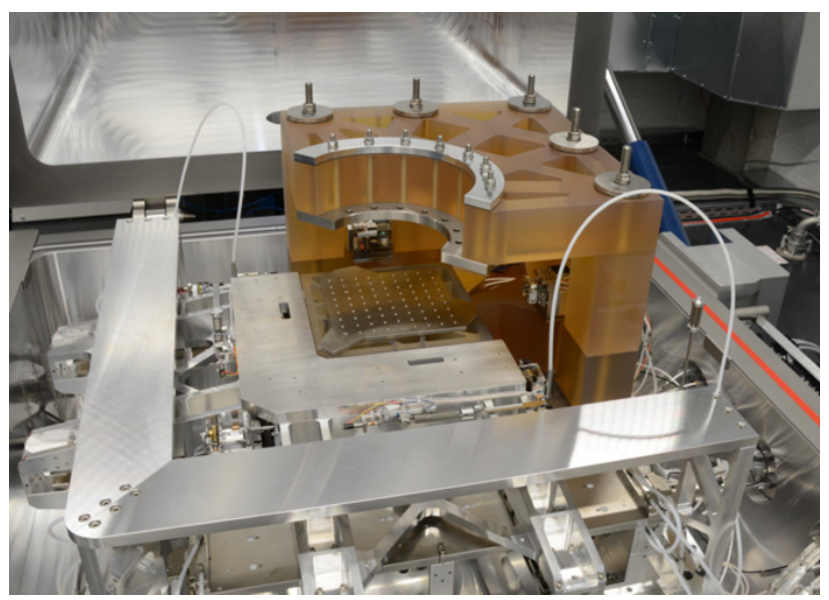

Abb. 6: Bild der NPMM-200 mit metrologischem Rahmen aus Zerodur $^{\circledR}$, Messobjektträger und magnetischen Linearantrieben.

Um höchste Positioniergenauigkeiten zu erreichen, arbeitet die Maschine in einer aktiv thermisch kontrollierten Vakuumkammer bei 1 mbar. Nach [19] können so Positionierunsicherheiten von weniger als $30 \mathrm{~nm}$ in einem Messvolumen von $200 \mathrm{~mm} \times 200 \mathrm{~mm} \times 25 \mathrm{~mm}$ erreicht werden.

\subsection{Messaufbau und Vorgehen}

Das Multi-Punkt-Positionsmesssystem besteht aus einem modifizierten telezentrischen Objektiv (Vico DTCM110-150-AL, $\left.N A=0,0085, \beta^{\prime}=-0,11\right)$ und einer CMOS-Kamera (Ximea MC023MG-SY, Sensorauflösung:

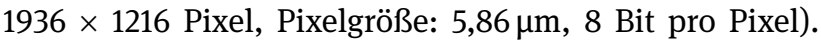
In der Aperturebene des Objektivs ist das lithografisch hergestellte DOE zur Erzeugung der Spotmuster angebracht. Zur Kalibrierung des Systems wurde eine LED $($ SMB1N-700, $\lambda=700 \mathrm{~nm}, \mathrm{HWB}=21 \mathrm{~nm}$, Strahlungsintensität $=40 \mathrm{~mW} / \mathrm{sr}$ ) mit unmittelbar davor angebrachter Kreisblende $(\mathrm{D}=200 \mu \mathrm{m})$ mäanderförmig in der NPMM200 an $M$ Messpunkte verfahren. Der Durchmesser jedes Einzelspots auf dem Sensor betrug etwa 12 Pixel, wobei nur Intensitäten unterhalb einer rauschabhängigen Schwelle berücksichtigt wurden. An jedem Messpunkt wird sowohl die Position der NPMM-200 $\left(X_{W}, Y_{W}\right)$ als auch die gemittelte Position $\left(x_{d}, y_{d}\right)$ des Spotmusters gespeichert. Die gemittelte Position des Spotmusters ergibt sich über eine Mittelung aller $N$ Einzelschwerpunkte.

Aufgrund der Ausdehnung des Spotmusters kann nicht die gesamte Sensorfläche der Kamera zur Positionsmessung genutzt werden. Es wurden nur Messpunkte ausgewertet, deren gemittelte Position mindestens 150 Pixel vom Rand des Detektors entfernt sind. Im Objektraum entspricht dies einem Messbereich von etwa $87 \mathrm{~mm} \times 49 \mathrm{~mm}$.

Bei den bisherigen Simulationen wurde von einer Verzeichnungsfunktion nach Gleichung (6) ausgegangen. Für die Anwendung als Messsystem ist jedoch der umgekehrte Weg nötig. Daher wurde für die folgenden Kalibriervorgänge die Position in Weltkoordinaten in Abhängigkeit von den gemessenen verzeichneten Koordinaten ausgedrückt

$$
\begin{aligned}
\tilde{X}_{W} & =\sum_{i=0}^{n} \sum_{j=0}^{n} L_{i j} x_{d}^{i} y_{d}^{j} \\
\tilde{Y}_{W} & =\sum_{i=0}^{n} \sum_{j=0}^{n} M_{i j} x_{d}^{i} y_{d}^{j}
\end{aligned}
$$

\subsection{Ergebnisse}

Die Kalibrierung wurde in 5 verschiedenen Z-Ebenen des NPMM-200-Koordinatensystems mit je $M>500$ Messpunkten durchgeführt. Die Ebenen liegen je $6 \mathrm{~mm}$ auseinander, beginnend bei $0,5 \mathrm{~mm}$ bis $24,5 \mathrm{~mm}$. Die Fehlerfunktion für die Least-Squares-Optimierung der Koeffizientenmatrizen $L_{i j}$ und $M_{i j}$ ergibt sich aus dem Abstand zwischen den von der NPMM-200 gemessenen Koordinaten $\left(X_{W}, Y_{W}\right)$ und den durch das Polynom bestimmten Koordinaten $\left(\tilde{X}_{W}\right.$, $\tilde{Y}_{W}$ ). Dieser Abstand entspricht auch dem Restfehler.

Die Verteilung des durchschnittlichen Restfehlers in der Ebene $Z=18,5 \mathrm{~mm}$ ist in Abbildung 7 dargestellt. Die Verzeichnung wird größtenteils gut durch das Polynom korrigiert. Lediglich im Zentrum des Sensors bleibt ein Bereich mit einem größeren Restfehler, der in allen 5 ZEbenen an derselben Stelle auftritt. Aktuell ist noch nicht abschließend geklärt, was zu diesem Fehlerbereich führt. 


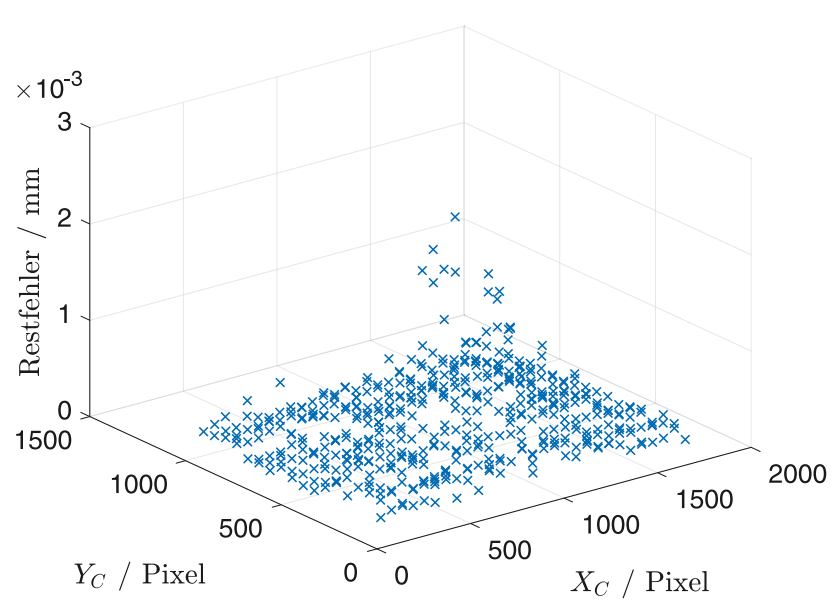

Abb. 7: Verteilung des absoluten Restfehlers im Objektraum bei $\mathrm{Z}=18,5 \mathrm{~mm}$, Ordnung $=9$.

Wir gehen davon aus, dass es sich um einen hochfrequenten Fehler handelt, der von einem Polynom 9. Ordnung nicht korrigiert werden kann. Als Fehlerquellen kommen unter anderem Schmutzpartikel auf dem Detektor oder Reflektionen zwischen den Objektivlinsen in Frage. Beide Einflüsse erzeugen eine Schwerpunktverschiebung und somit Sprünge in der Kalibrierlandschaft, die mit einer stetigen Funktion nur durch hohe Polynomordnungen erfasst werden können.

In Tabelle 2 sind die Restfehler im Objektraum für die Polynomordnungen 1 bis 9 in der Ebene $Z=18,5 \mathrm{~mm}$ aufgeführt. Die Ergebnisse können mit Hilfe der Objektivvergrößerung $\left|\beta^{\prime}\right|=0,11$ in den Bildraum projiziert werden. Die Standardabweichungen der bildseitigen Restverzeichnung liegen demnach bei $\sigma_{x}=0,030 \mu \mathrm{m}$ und $\sigma_{y}=0,034 \mu \mathrm{m}$, was in Pixeln ausgedrückt 5/1000 Pixel und 6/1000 Pixel. Diese Werte liegen erheblich unterhalb der beim Stand der Technik erzielten Kalibrierrestfehler (siehe Abschnitt 1.3).

Tab. 2: Restfehler im Objektraum in Ebene $Z=18,5 \mathrm{~mm}$.

\begin{tabular}{rrrrr}
\hline \multirow{2}{*}{ Ordnung } & \multicolumn{2}{l}{ Restfehler im Objektraum } & & \\
\cline { 2 - 5 } & Maximal & Durchschn. & $\boldsymbol{\sigma}_{\boldsymbol{x}}$ & $\boldsymbol{\sigma}_{\boldsymbol{y}}$ \\
\hline 1 & $23,64 \mu \mathrm{m}$ & $7,474 \mu \mathrm{m}$ & $4,828 \mu \mathrm{m}$ & $6,805 \mu \mathrm{m}$ \\
3 & $3,198 \mu \mathrm{m}$ & $0,985 \mu \mathrm{m}$ & $0,671 \mu \mathrm{m}$ & $0,890 \mu \mathrm{m}$ \\
5 & $2,864 \mu \mathrm{m}$ & $0,560 \mu \mathrm{m}$ & $0,460 \mu \mathrm{m}$ & $0,514 \mu \mathrm{m}$ \\
7 & $2,341 \mu \mathrm{m}$ & $0,370 \mu \mathrm{m}$ & $0,334 \mu \mathrm{m}$ & $0,371 \mu \mathrm{m}$ \\
9 & $1,986 \mu \mathrm{m}$ & $0,268 \mu \mathrm{m}$ & $0,248 \mu \mathrm{m}$ & $0,294 \mu \mathrm{m}$ \\
\hline
\end{tabular}

\section{Zusammenfassung}

Wir haben gezeigt, dass für eine hochgenaue Kamerakalibrierung $(<1 / 100$ Pixel) die gängigen Korrekturfunktionen nach dem physikalischen Modell nicht ausreichend genau sind. Der allgemeine Polynomansatz des phänomenologischen Modells lieferte in der Simulation eine erheblich bessere Korrektur des stark verzeichneten Linsensystems (etwa 1/1000 Pixel). Weitergehende Untersuchungen zur Kalibriermethode haben gezeigt, dass das oftmals verwendete Kalibriertarget nicht geeignet ist, da die zur Kalibrierung verwendete Lichtquelle der zur Messung eingesetzten in Größe und Abstrahlcharakteristik möglichst gleichen sollte. Die zur Kalibrierung benötigten Messdaten im Objekt- und Bildraum wurden mit Hilfe der NPMM200 erstellt. Eine Einzellichtquelle wurde mäanderförmig im Objektraum in 5 Z-Ebenen verfahren und an jedem Messpunkt sowohl die Maschinenposition als auch die gemittelten Position des Spotmusters gespeichert. Für jede Z-Ebene wurden durch eine Least-Squares-Optimierung die Kalibrierkoeffizienten ermittelt. Der durchschnittliche Restfehler liegt in allen 5 Ebenen bei unter 0,324 $\mu \mathrm{m}$, was im Bildraum einem Restfehler von unter 0,036 $\mu \mathrm{m}$ beziehungsweise 6/1000 Pixel entspricht.

Finanzierung: Wir danken der Deutschen Forschungsgemeinschaft (DFG) für die finanzielle Unterstützung des Projekts "Dynamische Referenzierung von Koordinatenmess- und Bearbeitungsmaschinen” (OS 111/42-2 und SA 847/16-2).

\section{Literatur}

1. Shaoqiong Wang, Bixi Yan, Mingli Dong, Jun Wang, and Peng Sun. An improved centroid location algorithm for infrared led feature points. In Sixth International Symposium on Precision Mechanical Measurements. SPIE, 2013.

2. Tobias Haist, Shihao Dong, Thomas Arnold, Marc Gronle, and Wolfgang Osten. Multi-image position detection. Optics express, 22:14450-14463, 062014.

3. Damien Douxchamps and Kunihiro Chihara. High-accuracy and robust localization of large control markers for geometric camera calibration. IEEE Trans. Pattern Anal. Mach. Intell., 31(2):376-383, 2009.

4. Dong Li and Jindong Tian. An accurate calibration method for a camera with telecentric lenses. Optics and Lasers in Engineering, 51(5):538-541, 2013.

5. Zhongwei Tang, Rafael Grompone von Gioi, Pascal Monasse, and Jean-Michel Morel. A precision analysis of camera distortion models. Trans. Img. Proc., 26(6):2694-2704, June 2017. 
6. Linshen Yao and Haibo Liu. A flexible calibration approach for cameras with double-sided telecentric lenses. International Journal of Advanced Robotic Systems, 13(3):82, 2016.

7. Flavio Guerra, Simon Hartlieb, Alexander Warsewa, Tobias Haist, Wolfgang Osten, and Oliver Sawodny. Deformation measurement of large buildings by holographical point replication. In Optics for Arts, Architecture, and Archaeology VII, volume 11058, pages 32-38. International Society for Optics and Photonics, SPIE, 2019.

8. Z. Zhang. A flexible new technique for camera calibration. IEEE Transactions on Pattern Analysis and Machine Intelligence, 22(11):1330-1334, Nov 2000.

9. Richard Hartley and Andrew Zisserman. Multiple View Geometry in Computer Vision. Cambridge University Press, New York, NY, USA, 2 edition, 2003.

10. Guan Banglei, Linshen Yao, HaiBo Liu, and Yang Shang. An accurate calibration method for non-overlapping cameras with double-sided telecentric lenses. Optik - International Journal for Light and Electron Optics, 131, 112016.

11. Junhua Sun, Xu Chen, Zheng Gong, Zhen Liu, and Yuntao Zhao. Accurate camera calibration with distortion models using sphere images. Optics \& Laser Technology, 65:83-87, 2015.

12. R. Usamentiaga, D. F. Garcia, C. Ibarra-Castanedo, and X. Maldague. Highly accurate geometric calibration for infrared cameras using inexpensive calibration targets. Measurement, 112:105-116, 2017.

13. R. Tsai. A versatile camera calibration technique for high-accuracy $3 \mathrm{~d}$ machine vision metrology using off-the-shelf tv cameras and lenses. IEEE Journal on Robotics and Automation, 3(4):323-344, August 1987.

14. Wolfgang Förstner and Bernhard P. Wrobel. Photogrammetric Computer Vision: Statistics, Geometry, Orientation and Reconstruction. Springer Publishing Company, Incorporated, 1st edition, 2016.

15. Juyang Weng, Paul Cohen, Marc Herniou, et al. Camera calibration with distortion models and accuracy evaluation. IEEE Transactions on pattern analysis and machine intelligence, 14(10):965-980, 1992.

16. Kepeng Chen, Tielin Shi, Wang Xiao, Yichun Zhang, Hong Yuan, Qiang Liu, and Guanglan Liao. Calibration of telecentric cameras with an improved projection model. Optical Engineering, 57:1, 042018.

17. Helmut Zollner and Robert Sablatnig. Comparison of methods for geometric camera calibration using planar calibration targets. In Digital Imaging in Media and Education, Proc. of the 28th Workshop, volume 179, page 237-244. Austrian Association for Pattern Recognition, 2004.

18. Joaquim Salvi, Xavier Armangué, and Joan Batlle. A comparative review of camera calibrating methods with accuracy evaluation. Pattern Recognition, 35(7):1617-1635, 2002.

19. Gerd Jäger, Eberhard Manske, Tino Hausotte, A. Müller, and F. Balzer. Nanopositioning and nanomeasuring machine NPMM-200-a new powerful tool for large-range microand nanotechnology. Surface Topography: Metrology and Properties, 4(3):034004, jul 2016.

20. Haifeng Xu. Hochpräzise Bestimmung der Form- und Orthogonalitätsabweichungen einer Spiegelecke und Untersuchung des Verhaltens unter veränderlichen Umweltbedingungen. PhD thesis, Ilmenau, Feb 2019.

\section{Autoreninformationen}

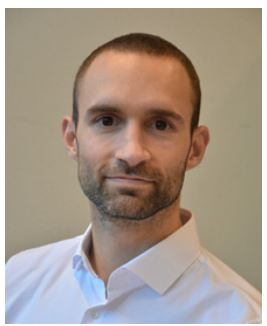

\author{
Simon Hartlieb \\ Institut für Technische Optik, Universität \\ Stuttgart, Pfaffenwaldring 9, 70569 \\ Stuttgart, Germany \\ hartlieb@ito.uni-stuttgart.de
}

Simon Hartlieb ist seit 2018 wissenschaftlicher Mitarbeiter am Institut für Technische Optik (ITO) der Universität Stuttgart. Von 2014 bis 2017 erlangte er den Abschluss M.Sc. in Maschinenbau mit Schwerpunkt auf Technische Optik und Mikrosystemtechnik an der Universität Stuttgart. Seine Hauptforschungsinteressen sind optische Positionsmessung, Bildverarbeitung und 3D Messtechnik.

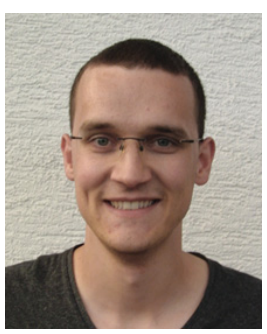

\section{Michael Tscherpel} Institut für Technische Optik, Universität Stuttgart, Pfaffenwaldring 9, 70569 Stuttgart, Germany tscherpel@ito.uni-stuttgart.de

Michael Tscherpel hat Photonic Engineering an der Universität Stuttgart studiert. Er ist seit Mai 2019 als wissenschaftlicher Mitarbeiter am Institut für Technische Optik (ITO) in der Arbeitsgruppe "3DMesstechnik" angestellt.

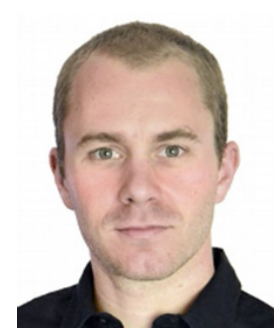

\section{Flavio Guerra} Institut für Technische Optik, Universität Stuttgart, Pfaffenwaldring 9, 70569 Stuttgart, Germany guerra@ito.uni-stuttgart.de

Flavio Guerra received the $M$. Sc. degree in photonic engineering from the University of Stuttgart, Germany, in July 2017. In August 2017, he joined the Institute of Applied Optics, University of Stuttgart, Germany, where he currently works as a research scientist and PhD student. His work focuses on 3D optical metrology and the application of multipoint technique to large scale objects. 


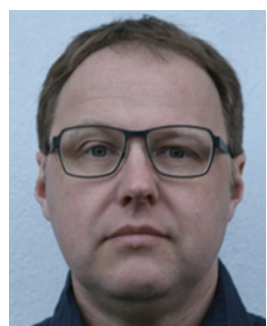

\section{Tobias Haist}

Institut für Technische Optik, Universität

Stuttgart, Pfaffenwaldring 9, 70569

Stuttgart, Germany

Haist@ito.uni-stuttgart.de

Tobias Haist hat Physik an der Universität Stuttgart studiert und dort im Bereich Technische Optik promoviert. Er ist akademischer Oberrat am Institut für Technische Optik (ITO) und Gruppenleiter der Arbeitsgruppe "3D Metrologie". Seine Hauptforschungsinteressen sind räumliche Lichtmodulation, 3D Messtechnik sowie Bildverarbeitung.

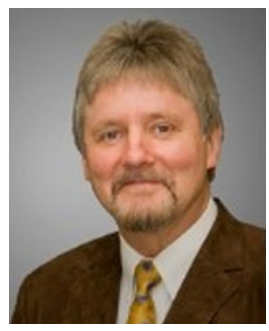

\section{Wolfgang Osten}

Institut für Technische Optik, Universität Stuttgart, Pfaffenwaldring 9, 70569

Stuttgart, Germany

osten@ito.uni-stuttgart.de

Prof. Dr. sc. nat. Wolfgang Osten war Direktor des Instituts für Technische Optik (ITO) der Universität Stuttgart u. a. mit den Hauptarbeitsgebieten: kohärente Optik, optische Messtechnik, digitale Bildverarbeitung, optische Sensorik für Produktionsautomatisierung, 3DOberflächenmesstechnik sowie kombinative optische Messverfahren.

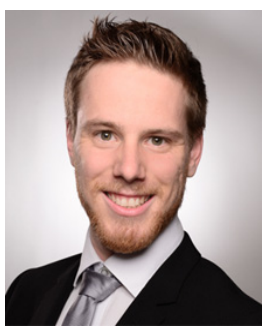

\section{Michael Ringkowski}

Institut für Systemdynamik, Universität Stuttgart, Waldburgstr. 19, 70563 Stuttgart, Germany

ringkowski@isys.uni-stuttgart.de

Michael Ringkowski received the B.Sc. and M.Sc. degrees in Engineering Cybernetics from the University of Stuttgart, Stuttgart, Germany, in 2014 and 2017, respectively. Since 2017 he has been a Research Assistant at the Institute for System Dynamics, University of Stuttgart, Germany. His current research interests include estimation and control of coordinate measuring machines, with a focus on increasing dynamics while maintaining precision.

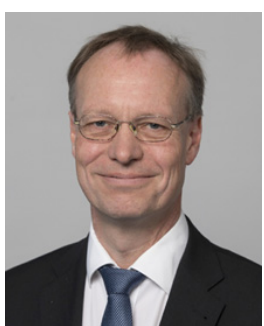

\section{Oliver Sawodny}

Institut für Systemdynamik, Universität Stuttgart, Waldburgstr. 19, 70563 Stuttgart, Germany

sawodny@isys.uni-stuttgart.de

Oliver Sawodny received the Dipl.-Ing. degree in electrical engineering from the University of Karlsruhe, Karlsruhe, Germany, in 1991, and the Ph.D. degree from the Ulm University, Ulm, Germany, in 1996. In 2002, he became a Full Professor with the Technical University of Ilmenau, Ilmenau, Germany. Since 2005, he has been the Director of the Institute for System Dynamics, University of Stuttgart, Stuttgart, Germany. His current research interests include methods of differential geometry, trajectory generation, and applications to mechatronic systems. 